\title{
MELHORIA DA QUALIDADE DE ENSAIOS DE AVALIAÇÃO DE CULTIVARES DE MILHO ATRAVÉS DE INDICADORES DE FERTILIDADE DO SOLO
}

\author{
THE USE OF SOIL FERTILITY DATA TO IMPROVE PRECISION OF MAIZE YIELD TRIALS
}

\author{
Sandra Feijó ${ }^{1}$ Lindolfo Storck $^{2}$ Alessandro Dal'Col Lúcio ${ }^{3}$ Cláudio Lovato $^{4}$ \\ Thomas Newton Martin ${ }^{5}$
}

RESUMO

O presente trabalho teve como objetivo verificar se o uso dos resultados da análise do solo numa análise da covariância com o rendimento de grão, aumenta a precisão experimental dos ensaios de avaliação de cultivares de milho. Os três ensaios constaram de 30 cultivares de ciclo precoce, 25 cultivares de ciclo superprecoce e nove cultivares de ciclo normal. Os ensaios foram realizados na área experimental do Departamento de Fitotecnia da UFSM. A adubação foi realizada para uma expectativa de rendimento maior que 6t.ha ${ }^{-1}$. A unidade experimental constituiu-se de duas filas de cinco metros de comprimento, espaçadas em oitenta centímetros, e o delineamento foi em blocos ao acaso com três repetições. As amostras de solo, seis subamostras tiradas na fila de semeadura, em cada unidade experimental, foram coletadas por ocasião do estádio de maturação fisiológica do milho. Verificou-se, com a análise da covariância, que o efeito do ajustamento das médias de rendimento de grãos com os indicadores de fertilidade não foi significativo. Conclui-se que a análise da covariância, utilizando os dados de análise de solo, não contribuiu para aumentar a precisão experimental da variável rendimento de grãos.

Palavras-chave: milho, covariância, precisão experimental.

\section{SUMMARY}

The objetive of this work was to verify whether the results of soil analysis in a covariance analysis of maize seed yield would increase the experimental precision of maize variety experiments. Three trials were conducted consisting of 30 early varieties, 25 considered as super early and nine as normal cycle. They were conducted at the Federal University of Santa Maria and the fertilizer levels were for a seed yield expectancy above six tons per hectare. The experimental units consisted of two rows of five meters length spaced $0.8 m$ in randomized block design with three replications. The soil samples consisted of six sub samples taken at maturity in the rows of each experimental unit. The results indicated that with a covariance analysis, the effect of adjusting the averages seed yields with the soil fertiity indicators was not significant and it was concluded that the covariance analysis did not contribute to increase the experimental precision of the variable seed yield.

Key words: maize, covariance, experimental precision.

\section{INTRODUÇÃO}

Vários trabalhos relatam que é baixa a precisão experimental dos ensaios de avaliação de cultivares de milho (LOPES, 1993; LÚCIO, 1997; LOPES \& STORCK, 1998; MARQUES, 1999). Isso implica erros (Tipo I e Tipo II) de conclusões ao se recomendar cultivares, pois as estatísticas são afetadas pelo aumento do erro experimental, podendo ocasionar recomendações errôneas de cultivares, determinando um uso indevido e obtenção de rendimentos inferiores e, como consequiência, redução na lucratividade da cultura. A qualidade de um experimento é avaliada pela magnitude do erro experimental; assim, os fatores que são potenciais fontes, que para ele podem contribuir, devem merecer uma atenção especial, pois afetam, em maior ou menor grau, a qualidade de um experimento (STORCK \& LOPES, 1997).

\footnotetext{
${ }^{1}$ Engenheiro Agrônomo, Mestre em Agronomia pela Universidade Federal de Santa Maria (UFSM). Bolsista da CAPES.

${ }^{2}$ Engenheiro Agrônomo, Doutor, Professor Titular do Departamento de Fitotecnia, UFSM, 97105-900, Santa Maria, RS. storck@ccr.ufsm.br (Autor para correspondência), Bolsista do CNPq.

${ }^{3}$ Engenheiro Agrônomo, Doutor, Professor Adjunto, Departamento de Fitotecnia, UFSM.

${ }^{4}$ Engenheiro Agrônomo, Doutor, Professor Titular, Departamento de Fitotecnia, UFSM.

${ }^{5}$ Acadêmico do Curso de Agronomia, UFSM. Bolsista do PIBIC/CNPq.
} 
É pouco provável que as condições físicas e químicas do solo sejam distribuídas aleatoriamente na área experimental, pois as parcelas adjacentes tendem a ser correlacionadas por fatores de ambiente (MARKUS, 1974). Uma das dificuldades encontradas nos ensaios de campo deve-se à dificuldade de observar solos em condições uniformes, mesmo tratando-se de superfícies reduzidas.

A heterogeneidade do solo, medida pelo rendimento das plantas cultivadas em pequenas parcelas, pode ser conseqüência da topografia do local, da umidade do solo, da variação na fertilidade ou de práticas agrícolas anteriores (HAYES \& IMMER, 1951). Em terrenos já adubados diversas vezes, o solo se torna mais heterogêneo (BASTOS, 1987). A heterogeneidade do solo é, talvez, a causa mais importante do erro experimental (LA LOMA, 1966) em que tais diferenças fazem com que os tratamentos apresentem resultados diferentes em cada repetição (MIRANDA FILHO, 1987). SIQUEIRA (1983) cita que solos pobres, com baixo nível de adubação, proporcionam menor variância que solos bem adubados. Também, a precisão experimental é menor em solos ácidos, com nível tóxico de alumínio, que em solos com pH e alumínio adequados à produção do milho (RESENDE, 1989).

A análise da covariância é assim denominada, porque se procede à análise da variância simultaneamente, para duas ou mais variáveis (SNEDECOR \& COCHRAN, 1967; STEEL $\boldsymbol{e}$ t al, 1997; GOMES, 1990). Geralmente, como resultado de um experimento, têm-se uma variável $(\mathrm{Y})$ dependente principal e uma ou mais variáveis $(\mathrm{X} 1, \mathrm{X} 2, \ldots)$ independentes secundárias, denominadas de covariáveis. Considera-se que essas covariáveis são correlacionadas entre si e com a variável principal e podem ser ou não dependentes dos tratamentos (de efeito fixo ou de efeito aleatório). A análise de covariância tem as suas aplicações, segundo STEEL $\boldsymbol{e t}$ al. (1997) nos seguintes casos: a) ajudar na interpretação dos dados, especialmente com relação à natureza dos efeitos de tratamentos; b) dividir uma covariância total ou soma de produtos nas partes componentes; c) controlar o erro e aumentar a precisão da medida dos efeitos de tratamentos; d) ajustar as médias de tratamentos da variável dependente, pelas diferenças nos correspondentes valores das variáveis independentes; e) estimar observações perdidas, através do método da pseudo-variável.

Quando a covariância é usada como um método de controle do erro, entende-se que a variação observada na variável dependente (Y) é, em parte, atribuída à variação nas covariáveis (X). Portanto, isso implica que a variação entre as médias (Y) é afetada pela variação entre as médias (X) de tratamentos, e que, para poder comparar os tratamentos, as médias $(\mathrm{Y})$ de tratamentos devem ser ajustadas de tal forma a torná-las as melhores estimativas, como as seriam se as médias $(\mathrm{X})$ de tratamentos fossem todas iguais. Similarmente, se o objetivo principal da covariância é ajustar as médias (Y) de tratamentos, então, obtém-se também um ajustamento do erro experimental (STORCK \& LOPES, 1997). BANZATTO \& KRONKA (1989) citam o uso da técnica de análise de covariância, a que, às vezes, pode ser usada para remover uma importante fonte de variação entre as unidades experimentais. STEEL $\boldsymbol{e t}$ al. (1997) relatam o uso da covariância para o controle do erro em dados obtidos em experimentos a campo e como um substituto para o uso de blocos.

KNAPP et al. (1995), em pesquisa de melhoramento para estresse por alumínio em áreas de avaliação de genótipos de milho, realizaram dois ensaios. No primeiro, constataram que a saturação de alumínio e zinco foi responsável por mais de 50\% da variação a campo entre 27 genótipos diferentes. No segundo ensaio, com 196 genótipos, realizaram uma análise de covariância usando os valores estimados de saturação de alumínio como covariante. Os resultados não mostraram nenhuma melhoria na eficiência estatística, quando comparados com médias não ajustadas.

As pressuposições necessárias para a validade da análise de covariância, segundo STEEL $\boldsymbol{e t}$ al. (1997), são: a) as observações Xij são de efeito fixo e medidas sem erro, independente do efeito de tratamentos; b) a regressão de Y sobre X, após removidos os efeitos de tratamentos e de blocos, é linear e independente dos tratamentos e de blocos. Assim, o coeficiente de regressão linear é homogêneo ( é o mesmo para cada tratamento); c) os erros são normal e independentemente distribuídos com média zero e variância comum $\sigma^{2}$

Para avaliar a eficiência da covariância, como método do controle do erro experimental, é feita uma comparação entre as estimativas das variâncias das médias de tratamentos ajustados e não ajustados para a covariável em questão (STORCK \& LOPES, 1997).

O presente trabalho teve como objetivo verificar se o uso da covariância do rendimento de grãos com indicadores de fertilidade do solo $(\mathrm{pH}$ em água, índice SMP, fósforo disponível, potássio trocável, matéria orgânica, concentração de alumínio, cálcio e magnésio, determinação de $\mathrm{H}+\mathrm{Al}$, capacidade de troca de cátions efetiva e capacidade de troca de cátions em pH 7,0, saturação de alumínio e saturação de bases) como covariáveis, aumentaria a precisão experimental dos ensaios de avaliação de 
cultivares de milho de ciclos superprecoce, precoce e normal.

\section{MATERIAL E MÉTODOS}

No período de outubro/98 a abril/99 foram conduzidos três ensaios de avaliação de cultivares de milho recomendadas para o Rio Grande do Sul. Os ensaios foram realizados no campus da Universidade Federal de Santa Maria. Segundo a classificação climática de Köeppen, o clima da região é do tipo "Cfa", isto é, um clima subtropical úmido (MORENO, 1961), e o solo pertencente à unidade de mapeamento São Pedro.

Para os três ensaios realizados, o delineamento experimental foi de blocos ao acaso com três repetições e a unidade experimental era formada por duas filas com cinco metros de comprimento, espaçadas em oitenta centímetros, desprezando $0,5 \mathrm{~m}$ nas extremidades das filas. Ensaio 1: 30 cultivares de milho recomendadas de ciclo precoce, semeadas para uma população de 55000 plantas.ha ${ }^{-1}$. Ensaio 2 : 25 cultivares de milho recomendadas de ciclo superprecoce, semeadas para uma população de 65000 plantas.ha ${ }^{-1}$. Ensaio 3: nove cultivares de milho recomendadas de ciclo normal, semeadas para uma população de 50000 plantas.ha ${ }^{-1}$. O manejo para condução dos ensaios seguiu as recomendações técnicas para a cultura do milho no Estado do Rio Grande do Sul (RECOMENDAÇÕES, 1998). A adubação na semeadura foi de $300 \mathrm{~kg}$.ha ${ }^{-1}$ da fórmula 5-20-20, para uma expectativa de rendimento acima de 6t.ha ${ }^{-1}$, seguindo as recomendações de Adubação e Calagem para os Estados do Rio Grande do Sul e Santa Catarina (ROLAS, 1989). A área, precedida com cultivo de aveia preta e dessecada com glifosate, 20 dias antes da instalação dos ensaios, foi sulcada com aplicação do adubo na linha, mediante o uso de máquina semeadora direta. Os blocos foram demarcados e a semeadura nas unidades experimentais foi procedida manualmente no dia 29/10/98, colocando duas sementes por cova, distanciadas em $22,7 \mathrm{~cm}, 19,2 \mathrm{~cm}$ e $25 \mathrm{~cm}$ nos ensaios de ciclos precoce, superprecoce e normal, respectivamente. O desbaste foi realizado, deixando uma planta por cova, visando à população prevista para cada ciclo. A adubação de cobertura foi dividida em duas partes, sendo aplicados, manualmente, $60 \mathrm{~kg} \cdot \mathrm{ha}^{-1}$ de uréia em cada aplicação nos dias 14/12/98 e 28/12/98.

As variáveis avaliadas foram: a) rendimento de grãos de milho a $13 \%$ de umidade; b) análise do solo nas unidades experimentais - as 192 unidades experimentais foram amostradas com a coleta de seis subamostras nas linhas de semeadura, dentro de cada unidade experimental, a uma profun- didade de 0 a $10 \mathrm{~cm}$, por ocasião da maturação fisiológica das cultivares de milho, sendo essas amostras submetidas à análise química no Laboratório de Análise de Solo da Universidade Federal de Santa Maria, para as determinações de $\mathrm{pH}$ em água, índice SMP, fósforo disponível, potássio trocável, matéria orgânica, concentração de alumínio, cálcio e magnésio, determinação de $\mathrm{H}+\mathrm{Al}$, capacidade de troca de cátions efetiva e capacidade de troca de cátions em pH 7,0, saturação de alumínio e saturação de bases, segundo os métodos descritos pela Rede Oficial de Laboratórios de Análise de Solo do Rio Grande do Sul e Santa Catarina (ROLAS, 1989).

Para análise da covariância da variável rendimento de grãos, foram utilizadas como covariáveis o pH, índice SMP, Fósforo (P), Potássio (K), matéria orgânica (MO), concentração de Alumínio (Al), Cálcio (Ca) e Magnésio (Mg), determinação de $\mathrm{H}+\mathrm{Al}(\mathrm{H}+\mathrm{Al})$, capacidade de troca de cátions efetiva (CTCe) e em pH 7,0 (CTC7), saturação de Alumínio (SAl) e bases (Sbases), segundo o modelo de análise da covariância múltiplo

$$
\mathrm{Y}_{\mathrm{ij}}=\mathrm{m}+\mathrm{t}_{\mathrm{i}}+\mathrm{b}_{\mathrm{j}}+\sum_{\mathrm{k}} \beta_{\mathrm{k}}\left(\mathrm{X}_{\mathrm{kij}}-\overline{\mathrm{X}}_{\mathrm{k}} . .\right)+\mathrm{e}_{\mathrm{ij}}
$$

em que: $Y_{\mathrm{ij}}$ é uma observação (variável dependente $\mathrm{Y})$ do tratamento i no bloco j; $\mathrm{X}_{\mathrm{kij}}$ é uma observação da covariável de ordem $\mathrm{k}=1,2, \ldots, 13$ na unidade experimental que recebeu o tratamento i no bloco j; $\mathrm{m}, \mathrm{t}_{\mathrm{i}}$, e $\mathrm{b}_{\mathrm{j}}$ são a média, o efeito de tratamento e de bloco, respectivamente; $\beta_{k}$ são os coeficientes de regressão lineares das 13 covariáveis; $\mathrm{e}_{\mathrm{ij}}$ é o erro experimental.

Para os cálculos, foi usado o pacote estatístico NTIA (EMBRAPA,1997) de acordo com a metodologia de SEARLE (1971). O teste para comparação de médias usado foi o teste de Tukey, em nível de 5\% de probabilidade de erro tipo I.

\section{RESULTADOS E DISCUSSÃO}

Os resultados da análise da covariância para rendimento de grãos com as covariáveis indicadoras de fertilidade do solo estão apresentados na tabela 1. Observa-se que não houve efeito significativo para as covariáveis nos ensaios de ciclos superprecoce e normal. No ensaio de cultivares de ciclo precoce, houve efeito significativo, em nível de 5\% de probabilidade de erro, para a covariável percentagem de saturação de bases (Sbases). O ajustamento das médias em função de covariáveis deve ser procedido somente quando todas as covariáveis do modelo forem significativos. Dessa forma, mesmo tendo efeito significativo para cultivares no ensaio 
Tabela 1 - Graus de liberdade (GL) e quadrado médio (QM) das fontes de variação (FV) bloco, cultivar, covariáveis do solo $\mathrm{pH}$, índice SMP (SMP), fósforo $(\mathrm{P})(\mathrm{mg} / \mathrm{L})$, potássio $(\mathrm{K})(\mathrm{mg} / \mathrm{L})$, matéria orgânica $(\mathrm{MO})(\mathrm{m} / \mathrm{V})$, concentração de alumínio $(\mathrm{Al})\left(\mathrm{cmol}_{\mathrm{c}} / \mathrm{L}\right)$, concentração de cálcio $(\mathrm{Ca})\left(\mathrm{cmol}_{\mathrm{c}} / \mathrm{L}\right)$, concentração de magnésio $(\mathrm{Mg})\left(\mathrm{cmol}_{\mathrm{d}} \mathrm{L}\right), \quad$ concentração de $\mathrm{H}+\mathrm{Al} \quad(\mathrm{H}+\mathrm{Al})$ $\left(\mathrm{cmol}_{\mathrm{C}} / \mathrm{L}\right)$, capacidade de troca de cátions efetiva $(\mathrm{CTCe})\left(\mathrm{cmol}_{\mathrm{d}} \mathrm{L}\right)$, capacidade de troca de cátions a pH 7,0 (CTC7) ( $\left.\mathrm{cmol}_{\mathrm{c}} \mathrm{L}\right)$, saturação de alumínio (SAl)(\%) e saturação de bases (SBases)(\%), erro experimental, média e coeficiente de variação (CV) para a variável rendimento de grãos $\left(\mathrm{t}_{\text {.ha }}{ }^{-1}\right)$ nos ensaios de ciclos superprecoce (SP), precoce $(\mathrm{P})$ e normal(N). Santa Maria, RS, 1999.

\begin{tabular}{|c|c|c|c|c|c|c|}
\hline \multirow[b]{2}{*}{ FV } & \multicolumn{2}{|c|}{ SP } & \multicolumn{2}{|r|}{$\mathrm{P}$} & \multicolumn{2}{|r|}{$\mathrm{N}$} \\
\hline & GL & QM & GL & QM & GL & QM \\
\hline$\overline{\text { Bloco }}$ & 2 & $2,166^{\mathrm{ns}}$ & 2 & $4,410^{*}$ & 2 & $0,212^{\mathrm{ns}}$ \\
\hline Cultivar & 24 & $1,149^{\mathrm{ns}}$ & 29 & $1,450^{*}$ & 8 & $1,045^{\mathrm{ns}}$ \\
\hline $\mathrm{pH}$ & 1 & $0,448^{\text {ns }}$ & 1 & $0,437^{\mathrm{ns}}$ & 1 & $1,866^{\mathrm{ns}}$ \\
\hline SMP & 1 & $0,017^{\mathrm{ns}}$ & 1 & $0,471^{\text {ns }}$ & 1 & $0,851^{\mathrm{ns}}$ \\
\hline $\mathrm{P}$ & 1 & $0,474^{\mathrm{ns}}$ & 1 & $1,132^{\mathrm{ns}}$ & 1 & $0,001^{\mathrm{ns}}$ \\
\hline K & 1 & $0,0004^{\mathrm{ns}}$ & 1 & $0,404^{\mathrm{ns}}$ & 1 & $0,520^{\mathrm{ns}}$ \\
\hline $\mathrm{MO}$ & 1 & $0,025^{\mathrm{ns}}$ & 1 & $0,512^{\mathrm{ns}}$ & 1 & $0,065^{\mathrm{ns}}$ \\
\hline $\mathrm{Al}$ & 1 & $2,244^{\mathrm{ns}}$ & 1 & $0,192^{\mathrm{ns}}$ & 1 & $1,735^{\mathrm{ns}}$ \\
\hline $\mathrm{Ca}$ & 1 & $0,032^{\text {ns }}$ & 1 & $0,209^{\mathrm{ns}}$ & 1 & $0,604^{\mathrm{ns}}$ \\
\hline $\mathrm{Mg}$ & 1 & $1,100^{\mathrm{ns}}$ & 1 & $0,115^{\text {ns }}$ & 1 & $0,445^{\text {ns }}$ \\
\hline $\mathrm{H}+\mathrm{Al}$ & 1 & $1,221^{\mathrm{ns}}$ & 1 & $0,440^{\mathrm{ns}}$ & 1 & $0,0003^{\text {ns }}$ \\
\hline CTCe & 1 & $0,225^{\mathrm{ns}}$ & 1 & $0,025^{\mathrm{ns}}$ & 1 & $0,506^{\mathrm{ns}}$ \\
\hline СТC7 & 1 & $0,474^{\mathrm{ns}}$ & 1 & $0,041^{\text {ns }}$ & 1 & $0,092^{\mathrm{ns}}$ \\
\hline SAl & 1 & $2,184^{\mathrm{ns}}$ & 1 & $0,638^{\mathrm{ns}}$ & 1 & $1,604^{\mathrm{ns}}$ \\
\hline Sbases & 1 & $0,313^{\mathrm{ns}}$ & 1 & $3,644^{*}$ & 1 & $1,752^{\mathrm{ns}}$ \\
\hline Erro & 35 & 0,842 & 45 & 0,349 & 3 & 0,334 \\
\hline Média & \multirow{2}{*}{\multicolumn{2}{|c|}{4,50}} & \multicolumn{2}{|c|}{5,17} & \multicolumn{2}{|c|}{4,42} \\
\hline CV\% & & & & & & \\
\hline \multicolumn{7}{|c|}{ Usando a covariável Sbases } \\
\hline Bloco & & & 2 & $4,452^{*}$ & & \\
\hline Cultivar & & & 29 & $1,617 *$ & & \\
\hline Sbases & & & 1 & $0,035 \mathrm{~ns}$ & & \\
\hline Erro & & & 57 & 0,414 & & \\
\hline Média & \multicolumn{6}{|c|}{5,17} \\
\hline $\mathrm{CV} \%$ & \multicolumn{6}{|c|}{12,4} \\
\hline
\end{tabular}

*efeito significativo pelo teste de $\mathrm{F}$ em nível de $5 \%$ de probabilidade de erro; ns efeito não significativo.

de ciclo precoce, houve efeito significativo, em nível de $5 \%$ de probabilidade de de ciclo precoce, não se procede o ajustamento das médias em função da covariável Sbases, sem antes recalcular a análise da covariância somente com essa covariável (Tabela 1). Observa-se que, isoladamente, a covariável Sbases não afetou o rendimento de grãos e, portanto, o ajustamento das médias de cultivares de ciclo precoce em função das médias de Sbases não é necessário. Sendo o efeito de cultivares de ciclo precoce significativo, em nível de $5 \%$ de probabilidade de erro, procedeu-se a comparação das médias não ajustadas pelo teste de Tukey (Tabela 2).

Verificou-se que a totalidade das variáveis indicadoras de fertilidade do solo, nos três
Tabela 2 - Rendimento médio de grãos de milho $\left(\right.$ th.ha $\left.^{-1}\right)$, para as cultivares de ciclo precoce, média geral e diferença mínima significativa pelo teste de Tukey em nível de $5 \%$ de probabilidade de erro (DMS). Santa Maria, 1999.

\begin{tabular}{|c|c|c|}
\hline Cultivar & t.ha ${ }^{-1}$ & \\
\hline DINA 766 & 6,34 & $a^{*}$ \\
\hline TORK & 6,31 & $\mathrm{a}$ \\
\hline AVANT & 6,14 & $a b$ \\
\hline DINA 566 & 6,10 & $a b$ \\
\hline PREMIUM & 6,06 & $\mathrm{abc}$ \\
\hline XL 214 & 6,03 & $a b c d$ \\
\hline XL 212 & 5,93 & $\mathrm{abcde}$ \\
\hline XL 340 & 5,89 & $\mathrm{abcde}$ \\
\hline SG 150 & 5,61 & $\mathrm{abcde}$ \\
\hline G 800 & 5,60 & $\mathrm{abcde}$ \\
\hline AG 5011 & 5,55 & $\mathrm{abcde}$ \\
\hline STAR & 5,35 & $a b c d e$ \\
\hline AG 5014 & 5,31 & $\mathrm{abcde}$ \\
\hline XL 345 & 5,27 & $a b c d e$ \\
\hline AG 8014 & 5,24 & $\mathrm{abcde}$ \\
\hline AS 523 & 5,13 & $a b c d e$ \\
\hline AG 122 & 5,04 & $\mathrm{abcde}$ \\
\hline Z 8474 & 5,03 & $\mathrm{abcde}$ \\
\hline DINA 1000 & 5,02 & $\mathrm{abcde}$ \\
\hline XL 215 & 5,01 & $\mathrm{abcde}$ \\
\hline AS 32 & 4,77 & $\mathrm{abcde}$ \\
\hline AS 3466 & 4,63 & $\mathrm{abcde}$ \\
\hline AG 303 & 4,62 & $a b c d e$ \\
\hline DINA 170 & 4,48 & $\mathrm{abcde}$ \\
\hline C 701 & 4,43 & $\mathrm{abcde}$ \\
\hline Z 8440 & 4,29 & $\mathrm{abcde}$ \\
\hline AGROM 2003 & 4,15 & $\mathrm{bcde}$ \\
\hline AGROM 2010 & 4,01 & $\mathrm{cde}$ \\
\hline CEP 304 & 3,98 & $\mathrm{de}$ \\
\hline AG 519 & 3,95 & $\mathrm{e}$ \\
\hline Média & \multicolumn{2}{|c|}{5,17} \\
\hline $\operatorname{DMS}(\%)$ & \multicolumn{2}{|c|}{39,6} \\
\hline
\end{tabular}

* Cultivares com médias não ligadas por mesma letra diferem pelo teste de Tukey em nível de $5 \%$ de probabilidade de erro.

ensaios, não interferiram (de forma linear) no rendimento de grãos. Os indicadores de fertilidade do solo não foram eficientes no sentido de reduzir o erro do ensaio de ciclo superprecoce, precoce e normal. Da mesma forma, KNAPP et al. (1995) também não encontraram nenhuma melhoria na eficiência estatística mediante o uso de apenas uma covariável (saturação de alumínio).

Várias podem ter sido as causas da não significância dos 13 indicadores de fertilidade sobre o rendimento de grão de milho. Uma pode ser o grande número de covariáveis, possivelmente relacionadas entre si, reduzindo o número de graus de liberdade do erro sem, contudo, reduzir a soma de quadrados do erro, implicando maior quadrado médio do erro ou não eficiência do uso da análise da covariância.

Observando os resultado da tabela 3, nota-se que, em alguns casos ( $\mathrm{pH}$, índice SMP, $\mathrm{Al}$ e 
Tabela 3 - Média e amplitude das variáveis do solo [pH, índice SMP (SMP), fósforo $(\mathrm{P})(\mathrm{mg} / \mathrm{L})$, potássio $(\mathrm{K})(\mathrm{mg} / \mathrm{L})$, matéria orgânica $(\mathrm{MO})(\mathrm{m} / \mathrm{V})$, concentração de alumínio $(\mathrm{Al})\left(\mathrm{cmol}_{\mathrm{d}} \mathrm{L}\right)$, concentração de cálcio $(\mathrm{Ca})\left(\mathrm{cmol}_{\mathrm{c}} \mathrm{L}\right)$, concentração de magnésio $(\mathrm{Mg})\left(\mathrm{cmol}_{\mathrm{c}} / \mathrm{L}\right)$, concentração de $\mathrm{H}+\mathrm{Al} \quad(\mathrm{H}+\mathrm{Al}) \quad\left(\mathrm{cmol}_{\mathrm{c}} / \mathrm{L}\right)$, capacidade de troca de cátions efetiva $(\mathrm{CTCe})\left(\mathrm{cmol}_{\mathrm{c}} / \mathrm{L}\right)$, capacidade de troca de cátions a $\mathrm{pH} 7,0(\mathrm{CTC})\left(\mathrm{cmol}_{\mathrm{c}} / \mathrm{L}\right)$, saturação de alumínio (SAl)(\%) e saturação de bases (Sbases) $(\%)$ nos ensaios de ciclos superprecoce, precoce e normal. Santa Maria, RS, 1999.

\begin{tabular}{lcccccc}
\hline & \multicolumn{2}{c}{ Super precoce } & \multicolumn{2}{c}{ Precoce } & \multicolumn{2}{c}{ Normal } \\
\cline { 2 - 7 } Variável & Média & Amplitude & Média & Amplitude & Média & Amplitude \\
\hline pH & 4,95 & 0,40 & 4,81 & 0,70 & 4,95 & 0,40 \\
SMP & 5,33 & 0,60 & 5,35 & 0,70 & 5,29 & 0,50 \\
P & 4,4 & 27,0 & 16,7 & 58,8 & 24,5 & 62,0 \\
K & 87,6 & 136,4 & 130,4 & 120,0 & 104,9 & 120,0 \\
MO & 2,59 & 1,40 & 2,81 & 0,70 & 2,65 & 0,90 \\
Al & 0,84 & 2,50 & 0,87 & 1,20 & 0,81 & 1,40 \\
Ca & 4,40 & 2,70 & 4,20 & 3,20 & 4,23 & 2,20 \\
Mg & 1,85 & 1,10 & 1,70 & 1,30 & 2,11 & 1,00 \\
H+Al & 6,55 & 3,70 & 6,40 & 4,10 & 6,81 & 3,20 \\
CTCe & 7,3 & 4,0 & 7,1 & 4,5 & 7,4 & 2,4 \\
CTC7 & 13,0 & 4,3 & 12,6 & 5,0 & 13,4 & 3,1 \\
SAl & 11,5 & 25,0 & 12,4 & 19,0 & 11,1 & 23,0 \\
Sbases & 49,7 & 23,0 & 49,5 & 25,0 & 49,3 & 20,0 \\
\hline
\end{tabular}

SAl), o indicador de fertilidade foi insuficiente para a cultura, e a amplitude de variação foi baixa, não atingindo o nível ideal para a cultura. Em outros casos $(\mathrm{P}, \mathrm{K}, \mathrm{MO}, \mathrm{Ca}, \mathrm{Mg})$, o indicador foi suficiente e a amplitude, nesse caso, não foi um fator limitante para a cultura.

Segundo LOPES (1993), a heterogeneidade do solo pode ser amenizada em uma condição de maior fertilidade. Conforme a adubação utilizada nesses ensaios, pode-se comprovar que a diferença dos elementos indicadores da fertilidade do solo não tiveram uma amplitude de variação, que implicasse diferenças de fertilidade entre unidades experimentais, resultando em uma adubação eficiente, ou seja, uniforme, mesmo concluindo-se que a área era heterogênea antes da instalação dos ensaios, não sendo mais uma fonte de erro nos ensaios.

O ano agrícola de 98/99 foi um ano de produtividade média a baixa, o que implicaria média ou baixa precisão, conforme resultados de RESENDE (1989). Nesses casos, a covariância de rendimento de grãos com indicadores de fertilidade do solo deveria ser uma alternativa adequada para melhoria da precisão. $\mathrm{O}$ manejo da cultura não previu irrigação para permitir a condição adequada para o estudo do efeito das covariáveis. No caso, a covariância não foi eficiente para a redução do erro experimental.

Outro fator que pode ser causa da ineficiência do uso da análise da covariância é a represen- tatividade das amostras coletadas. Sob condições de estresse hídrico, as plantas de milho aprofundam suas raízes para captação de água e nutrientes em camadas mais profundas; a coleta de amostras de solo procedida foi realizada na profundidade de 0 a $10 \mathrm{~cm}$, o que pode não ser representativo da unidade experimental com relação à concentração de nutrientes realmente presentes. Ainda, há possibilidade de uma homogeneidade na fertilidade da área experimental, não permitindo a estimativa de uma relação com o rendimento de grão. Uma outra possível explicação é que outros fatores ambientais, que não a fertilidade do solo, foram os fatores que limitaram a produtividade aos patamares obtidos.

As cultivares de ciclo precoce apresentaram uma média geral igual a 5,17.ha ${ }^{-1}$ (Tabela 2). A cultivar DINA 766 obteve o maior rendimento $\left(6,34\right.$ t.ha $\left.{ }^{-1}\right)$ e o menor rendimento foi da cultivar AG $519\left(3,95\right.$ th.ha $\left.^{-1}\right)$. A diferença mínima significativa pelo teste de Tukey (DMS) obtida para este ensaio foi de $39,6 \%$, representa uma diferença entre cultivares de aproximadamente 2 t.ha ${ }^{-1}$, que é muito alta para a cultura do milho, refletindo baixa precisão. Esse valor da DMS é classificado, segundo LÚCIO (1997), como médio. No entanto, a grande amplitude de variação de produtividade entre as cultivares permitiu ainda a discriminação entre as mesmas.

Embora com rendimentos superiores ao da média do estado, a expectativa era para um rendimento ainda superior aos obtidos. Inúmeras são as causas que podem ter afetado o rendimento dos ensaios, entre elas a limitação hídrica é um fator crucial para o desempenho da cultura. As consequiências do estresse hídrico dependem de vários fatores, a saber: sanidade da planta, nível nutricional, estádio de desenvolvimento, duração, intensidade, genótipo. Ainda, o efeito da disponibilidade de nutrientes também ficou prejudicado em função do estresse hídrico, e LOPES (1993) também descreveu o mesmo motivo para o rendimento inferior das cultivares empregadas em seu experimento, usando adubações com expectativas superiores às obtidas.

O sistema de cultivo utilizado também pode ter afetado o rendimento de grãos de milho, sendo que vários autores estudaram o efeito do sistema de cultivo sobre o rendimento de grãos, em que plantas de milho cultivadas em sucessão a gramíneas absorvem menos $\mathrm{N}$ em relação às que se desenvolvem em sucessão a leguminosas, refletindo-se em menor rendimento de grãos. ARGENTA \& SILVA 
(1999) verificaram uma redução no rendimento de grãos de milho em sucessão ao cultivo de aveia preta, principalmente em sistema de plantio direto recentemente implantado.

\section{CONCLUSÃO}

O uso da análise da covariância do rendimento de grãos de milho com indicadores de fertilidade do solo ( $\mathrm{pH}$ em água, índice SMP, fósforo disponível, potássio trocável, matéria orgânica, concentração de alumínio, cálcio e magnésio, determinação de $\mathrm{H}+\mathrm{Al}$, capacidade de troca de cátions efetiva e capacidade de troca de cátions em $\mathrm{pH} 7,0$, saturação de alumínio e saturação de bases) não melhorou a precisão experimental nos ensaios de competição entre cultivares recomendadas de milho de ciclo superprecoce, precoce e normal.

\section{REFERÊNCIAS BIBLIOGRÁFICAS}

ARGENTA, G., SILVA, P.R.F. Adubação nitrogenada em milho implantado em semeadura direta após aveia preta. Ciência Rural, Santa Maria, v.29, n.4, p.745-754, 1999.

BANZATTO, D.A., KRONKA, S.N. Experimentação agrícola. Jaboticabal : FUNEP, 1989. 247p.

BASTOS, E. Guia para o cultivo do milho. São Paulo : Icone, 1987. $190 \mathrm{p}$

EMBRAPA. Empresa Nacional de Pesquisa Tecnológica em Informática para a Agricultura (Campinas-SP). Ambiente de software NTIA. Versão 4.2.2: manual do usuário ferramental estatístico. Campinas, 1997. 258p.

GOMES, F.P. Curso de estatística experimetal. 13 ed Piracicaba : Nobel, 1990. 468p.

HAYES, H.K., IMMER, F.K. Metodos fitotecnicos: procedimientos cientificos para mejorar las plantas cultivadas. 3 ed. Buenos Aires : Acme Agency, 1951. 560p.

KNAPP, E.B., CEBALLOS, H.C., PANDEY, S. Uso de análise espacial em áreas de avaliação para estresse de nutriente em milho. In: SIMPÓSIO INTERNACIONAL SOBRE ESTRESSE AMBIENTAL: o milho em perspectiva, 1, 1992, Belo Horizonte, MG. Anais... Sete Lagoas: EMBRAPA/CNPMS, 1995. 449p. p.97-106.

LA LOMA, J.L. Experimentación agrícola. 2 ed. México : UTEHA, 1966. 493p.

LOPES, S.J. Avaliação do efeito de diferentes formas de adubação sobre a precisão de ensaios de milho. Santa Maria - RS, 1993. 72p. Dissertação (Mestrado em Agronomia) - Curso de Pós-graduação em Agronomia Universidade Federal de Santa Maria, 1993
LOPES, S.J., STORCK, L. Heterogeneidade do solo sob diferentes adubações na cultura do milho. Ciência Rural, Santa Maria, v.28, n.3, p.361-366, 1998.

LÚCIO, A.D. Parâmetros da precisão experimental das principais culturas anuais do Estado do Rio Grande do Sul. Santa Maria - RS, 1997. 64p. Dissertação (Mestrado em Agronomia) - Curso de Pós-graduação em Agronomia, Universidade Federal de Santa Maria, 1997.

MARKUS, M. Elementos de estatística aplicada. Porto Alegre : UFRGS / Departamento de Estatística, 1974. 329p.

MARQUES, D.G. As pressuposições e a precisão dos ensaios de competição de cultivares de milho no Estado do Rio Grande do Sul. Santa Maria - RS, 1999. 42p. Dissertação (Mestrado em Agronomia) - Curso de Pós-graduação em Agronomia, Universidade Federal de Santa Maria, 1999.

MIRANDA FILHO, J.B. Melhoramento e produção do milho. Campinas : Fundação Cargill, 1987. V.2: Princípios de experimentação e análise estatística: p.765-794.

MORENO, J.A. Clima do Rio Grande do Sul. Porto Alegre : Secretaria da Agricultura, Diretoria de Terras e Colonização, Seção de Geografia, 1961. 46p.

RECOMENDAÇÕES técnicas para a cultura de milho no Estado do Rio Grande do Sul. Porto Alegre : FEPAGRO; EMATER; FECOAGRO, 1998. 147p. (Boletim Técnico, n.5).

RESENDE, M.D.V.de. Seleção de genótipos de milho (Zea mays L.) em solos contrastantes. Piracicaba - SP, 1989. 212p. Dissertação (Mestrado em Melhoramento de Plantas) Curso de Pós-graduação em Melhoramento de Plantas, Universidade de São Paulo, 1989.

ROLAS - Recomendações de adubação e calagem para os Estados do Rio Grande do Sul e Santa Catarina. 2 ed. Passo Fundo : SBCS - Núcleo Regional Sul/EMBRAPA CNPT, 1989. 128p.

SANTOS, R.F. Comportamento morfo-fisiológico de plantas de milho submetidas a deficit hídrico em solos de diferentes texturas. S anta Maria - RS, 1997. 90p. Dissertação (Mestrado em Agronomia) - Curso de Pósgraduação em Agronomia, Universidade Federal de Santa Maria, 1997.

SEARLE, S.R. Linear models. New York : John Wiley, 1971. $532 \mathrm{p}$.

SIQUEIRA, A.L. Uso da transformação em análise de variância e análise de regressão. São Paulo - SP, 1983. 154p. Dissertação (Mestrado em Estatística) - Curso de Pósgraduação em Estatística, Universidade de São Paulo, 1983.

SNEDECOR, G.W., COCHRAN, W.G. Statistical methods. 6 ed. Ames : Iowa State University, 1967. 593p.

STEEL, R.G.D., TORRIE, J.H., DICKEY, D. Principles and procedures of statistics: a biometrical approach. 3 ed. Boston : WCB/McGraw Hill, 1997. 666p.

STORCK, L., LOPES, S.J. Experimentação II. Santa Maria : UFSM/Departamento de Fitotecnia, 1997. 197p. 